\title{
Network Design for Cylinder Gas Distribution
}

\author{
Tejinder Pal Singh, Nicoleta Neagu, Michele Quattrone, Philippe Briet
}

Air Liquide (United States)

tejinder.singh@airliquide.com,nicoleta.neagu@airliquide.com, Michele.Quattrone@airliquide.com,Pbilippe.Briet@airliquide.com

\section{Abstract:}

Purpose: Network design of the supply chain is an important and strategic aspect of logistics management. In this paper, we address the network design problem specific to packaged gases (cylinder) supply chain. We propose an integrated framework that allows for the determination of the optimal facility locations, the filling plant production capacities, the inventory at plants and hubs, and the number of packages to be routed in primary and secondary transportation.

Design/methodology/approach: We formulate the problem as a mixed integer program and then develop a decomposition approach to solve it. We illustrate the proposed framework with numerical examples from real-life packaged gases supply chain. The results show that the decomposition approach is effective in solving a broad range of problem sizes.

Findings: The main finding of this paper is that decomposing the network design problem into two sub-problems is very effective to tackle the real-life large scale network design problems occurring in cylinder gas distribution by optimizing strategic and tactical decisions and approximating the operational decisions. We also benchmark the results from the decomposition approach by solving the complete packaged gases network design model for smaller test cases.

Originality/value: The main contribution of our work is that it integrates supply chain network design decisions without fixing the fillings plant locations with inventory and resource allocation decisions required at the plants. We also consider the transportation costs for the 
entire supply chain including the transhipment costs among different facilities by deciding the replenishment frequency.

Keywords: network design, optimization, location-routing, packaged gases, inventory management, decomposition approach, heuristics, mixed integer linear programming

\section{Introduction}

Supply chain networks are essential within the world wide economic activities. They are fundamental to stay competitive in today's markets through efficient delivery of products (e.g., energy, food, pharmaceutics, and clothing). The optimal supply chain network design is the basis for its efficiency. Moreover, the network design is a complex topic as it needs to take into account and integrate many aspects of real life problems.

In this paper we consider the packaged gases supply chain with its specific characteristics. Network configuration in packaged gases (also referred as cylinder distribution) is a strategic decision that impacts the tactical delivery planning and daily scheduling and transportation operations. A typical cylinder supply chain network consists of filling plants, hubs/distribution centres, and customers. Filling plants supply cylinders to hubs which distribute them to customers. It is also possible for filling plants to directly supply the customers. Filling plants and hubs manage cylinder stocks in order to enable the supply chain to maintain an adequate service level. The agility of the supply chain and the operational efficiency are constrained by the structure of the network determining the flow of material.

Optimizing the network design problem for cylinder distribution consists of determining the locations for filling plants/hubs, the production tools to be installed at the plants, the primary and the secondary flows, as well as the inventory at plants and customer locations. The framework based on a mixed-integer linear programming (MILP) model is developed to capture a real-life packaged gases business model. The mathematical model contains constraints on network structure, primary transportation, i.e., flow of cylinders among different supplier locations, secondary transportation, i.e., flow of cylinders from supplier locations to customers, stock management and assets management. The proposed framework has been developed by leveraging the best practices and knowledge of logistics experts within packaged gases supply chain. Therefore, the framework can be used to determine a new cylinder supply chain/logistics network for a new market or to study the impact of change in different elements of the supply chain, e.g., when new customer accounts are opened or old accounts are closed, change in customer demand, impact of new filling centres/hubs and assets like filling tools and manpower. We show the efficiency of the proposed framework for real-life test cases provided by the packaged gases supply chain managers. 
The paper is organized as follows. Section 2 outline the state of the art related to integrated supply chain decision models. In Section 3, we provide the problem description and in Section 4 we represent the mathematical model with the objective function and the business constraints. In Section 5, we discuss the solution approaches used to solve the integrated model. Section 6 presents the obtained results and Section 7 concludes the paper with possible future research directions.

\section{Literature Review}

The network design problem in packaged gases consists of three main sub-problems:

- Facility location: It involves the improvement of the existing network and the determination of the best configuration.

- Inventory management: It consists of determining the best inventory levels at hubs/plants.

- Routing: Optimization of flows determining the optimal flows of goods through the network.

The network design problems are complex as they involve strategic decisions which influence tactical and operational decisions (Crainic \& Laporte, 1997). The strategic decisions are mainly related to facility locations, their capacities and what products need to be produced at each plant, etc. The tactical decisions are related to inventory management and manpower, and depend on the strategic decisions whereas operational decisions like routing are directly related to tactical and strategic decisions made earlier. In other words, it means that if facility location decisions are sub-optimal, even if production, inventory and distribution plans are fully optimized, the supply chain may still be operating inefficiently. Therefore, for determining the best network configuration, all the costs at the three levels need to be taken into account to optimize the system-wide production, inventory and distribution costs. One of the challenges in the network configuration is that customer demands and cost parameters may change over time and it is very hard to change the facility location decisions once a supply chain network is configured. Thus, it is critical to design a supply chain network that is optimal and is not sensitive to changes in the operational parameters. The integrated network design problem has been usually solved by considering the integration of two sub-problems while approximating the third one. We provide following few approaches in the literature for solving the integrated network design problem.

The facility location problem integrated with routing is proved to be NP-complete by Krarup and Pruzan (1983). The objective function and the constraints of the models they propose are linear. The reader is referred to the reviews provided by Klose and Drexl (2005) and ReVelle 
and Eislet (2005). The facility location problem and its variants have been widely researched on theoretical models but the problem is rarely approached from a supply chain management and real-life perspective (Melo, Nickel \& Saldanha-da-Gama, 2009).

Most of the papers in the literature study the integration of two of the above three important decisions: location-routing models (LR), inventory-routing models, and location-inventory (LI) models. For reviews on location-routing models, readers can refer to Balakrishnan, Ward and Wong (1987). In LR models, both the location problem and the vehicle routing problem (VRP) are typically NP-hard, which makes the integrated model even more complex. In this paper, the VRP problem is solved by approximating the routes based on either a heuristic approach or historical data. The resulting routing costs are then fed as an input to the location model. For inventory-routing models, please refer to Kleywegt, Nori and Savelsbergh (2002) and Adelman (2003). LI models also study the location, inventory and distribution coordination issues by either ignoring the inventory costs or approximating the non-linear costs with linear functions. In this paper, inventory costs are considered but assumed to be linear similar to some papers that consider inventory costs. Refer to the papers Daskin and Owen (1999), Shen (2000), and Erlebacher and Meller (2000) for a better understanding of LI models.

The case that motivated this research deals with the network design for packaged gases distribution. The problem addressed in the current paper combines some elements of LR and LI models to determine an optimal network design by minimizing the sum of the production costs, the transportation costs and the inventory costs. Our mathematical model can be classified as a deterministic single-period MILP model with multiple products applied to a three-level network. The main contribution of our work is that it integrates supply chain network design decisions without fixing the fillings plant locations with inventory and resource allocation decisions required at the plants. We also consider the transportation costs for the entire supply chain including the transhipment costs among different facilities by deciding the replenishment frequency.

\section{Problem Description}

We address the network design problem specific to packaged gases supply chain occurring in real-life. The problem consists of determining the number and the location of the production plants and the distribution centres, the allocation of customer demands to distribution centres, and the allocation of distribution centres to production plants. The main goal is to identify the optimal configuration for producing and delivering packaged gas products to customers at the lowest cost while satisfying the network constraints.

More specifically, this network design problem aims in helping the decision making on locations for building plants, the production tools to be installed at the filling plants, the primary and the 
secondary flows, as well as the inventory at plants and hubs. A diagram of the packaged gases distribution network is shown in the Figure 1 . The nodes of the network are classified in four categories: filling plants, hubs, agent distributors, and end users (or reseller). Each location has a certain inventory capacity to satisfy customer demand. Customers manage their own inventories by placing orders at the right time. Therefore, in the current problem we consider the inventory decisions only at filling plants and hub locations.

The arrows in Figure 1 represent the transport of packaged gases which is classified as:

- Primary transport which occurs between filling plants and hub locations.

- Secondary transport which represents the transport between hub/filling plants and client/agent/reseller locations.

- Tertiary transport which happens between agent/reseller and client locations.

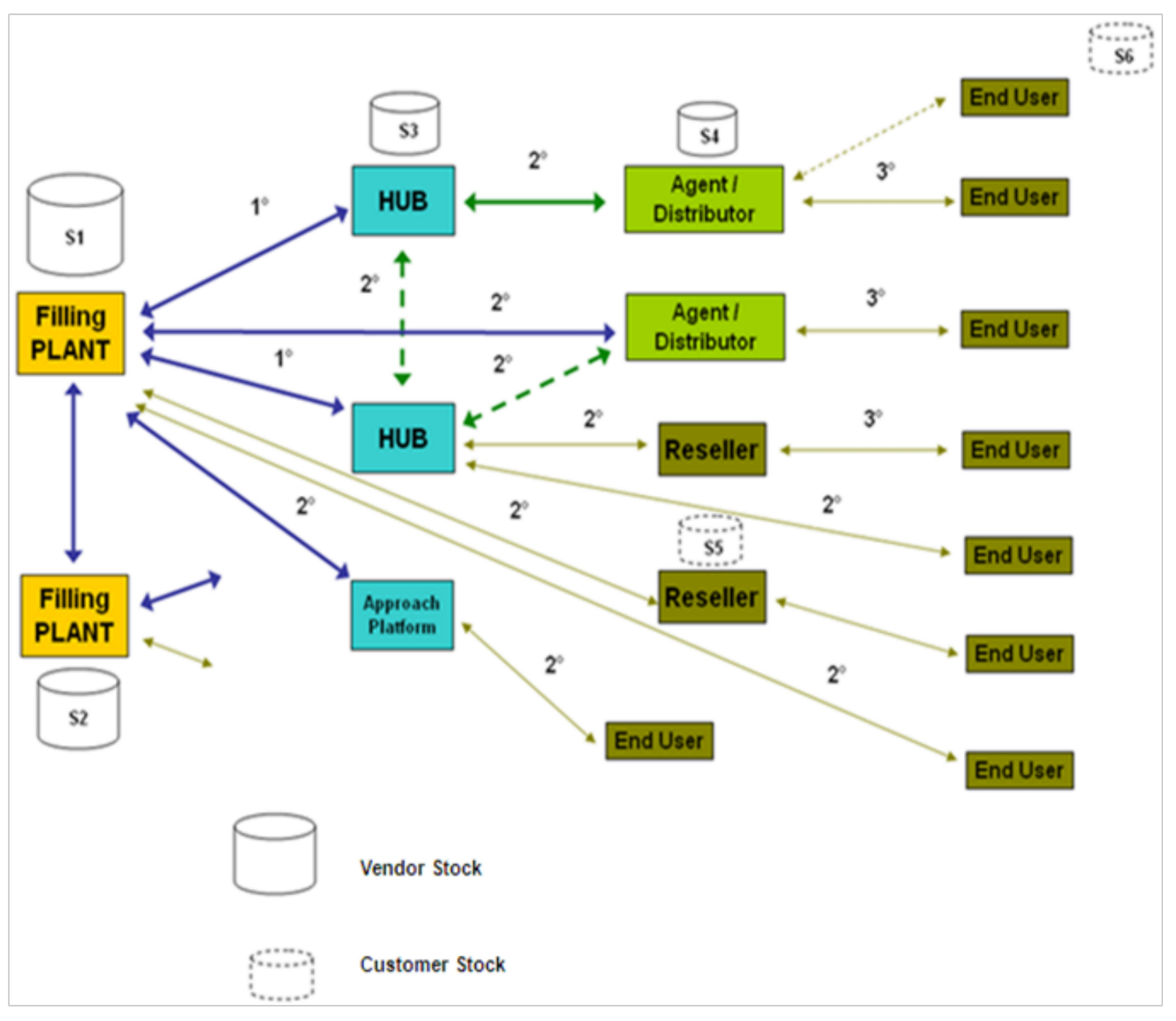

Figure 1. Diagram of the Distribution Network 
This paper will not handle the whole distribution network but rather will focus on the primary and secondary transport. Agents/distributors, resellers and end users will all be called customers without distinction in the rest of the paper. Since tertiary flows happen between customers of different types, they are not considered in this problem. The word "plant" by itself is referring to both hubs and filling plants. In this paper we also assume that the vendor who supplies cylinders to the customers owns the whole packaged gases supply chain network. Therefore, we do not consider any ordering costs between different plants. We do consider the transportation cost of transhipments among different plants which is a function of replenishment periods for the primary flows. The main goals of the proposed methodology are related to the location decisions of plants, production, and to the hubs/filling plants transport and inventory. The primary goals of the network design problem for packaged gases are as follows, see also Figure 2:

- Determine the number and the locations of the hubs and the filling plants.

- Determine the production of different products at the filling plants.

- Determine the primary and the secondary transportation cylinder flows, i.e. the customer-plant allocation decisions.

- Determine the inventory levels at plants consisting of working stock \& the safety stocks at the plants.

These four issues are fundamental in the structure of a supply chain. Nevertheless, these issues are interrelated by the cylinders flows and it is clear that it would be a source of improvement to treat them all at the same time. A general description of all the key aspects of the problem as well as the hypotheses assumed at this stage is presented in the following subsections.

\subsection{Multi-Products Network}

We assume that different products may be considered while designing the distribution network. This means that different products are filled at a plant, and transported to others plants and customers. Therefore, each product has to be characterized depending on its package, its composition and the filling tools that are compatible with it. 


\subsection{Plant Locations and Filling Tools}

The network design problem considers as input a set of potential locations already identified. A hub or a filling plant may be built on a location if selected by the optimizer. The total amount of cylinders distributed to the customers are filled on the filling plants and delivered from the hubs (or the plants). The maximum numbers of plants in the network is a function of the number of the potential locations provided in the input.

A product is defined as a cylinder of a given size filled with different gases in various percentages. The products are filled with gases using filling tools. Several different tools may be used at a filling plant; each of them allows filling a given set of products and has its own production capacity and cost.

\subsection{Cylinder Flows}

Two categories of cylinder flows are considered in this problem: primary and secondary. These two categories find their differences on two levels:

- Primary flows are an internal choice of optimization of the distribution without direct income. They usually go from one vendor site to another and require handling during the round trip: a tractor pulls a trailer of full products from site $A$ to site $B$, leaves the trailer on site $B$ and takes back a trailer of empty products from site $B$ to site $A$.

- Secondary flows are a direct source of income for the vendor as customers have to pay for the delivered cylinders. A secondary round trip is usually composed with several drops on different customers' sites where full products have to be unloaded before empty products are loaded on the trailer.

The transport cost is usually composed of a fixed cost and a variable cost. The fixed cost consists of truck costs, driver costs, and extra fees and the variable cost is dependent on the distance and the duration of the round trips. However, we model the cost of a round trip with an average variable cost per driver distance taking every cost into consideration.

The handling cost of the cylinders on plants is taken into consideration independently. In this paper, we are approximating the routing costs to serve each customer and therefore, do not consider the routing decisions in the model. 


\subsection{Primary Transport Cost}

The primary transport cost is quite straightforward to estimate. As primary trips are defined as full trailer load deliveries in the model, the cost of primary round trips between two identified plants can be known before solving the network design.

\subsection{Secondary Transport Cost}

Contrary to the primary transport, secondary transport cost is difficult to estimate precisely. In the network design model, we do not consider day-to-day demand data which implies that it is impossible to create actual secondary round trips. In the model, each customer has a global demand over a year and it is not possible to know which customers will order on the same day. Also, secondary unit transport costs are provided as an input to the problem and therefore, the actual costs can not be calculated until the plant-customer allocation decisions are made. Therefore, we determine the cost to deliver a unit cylinder to a customer from each potential location by modelling the average round trips during which the customer will be delivered.

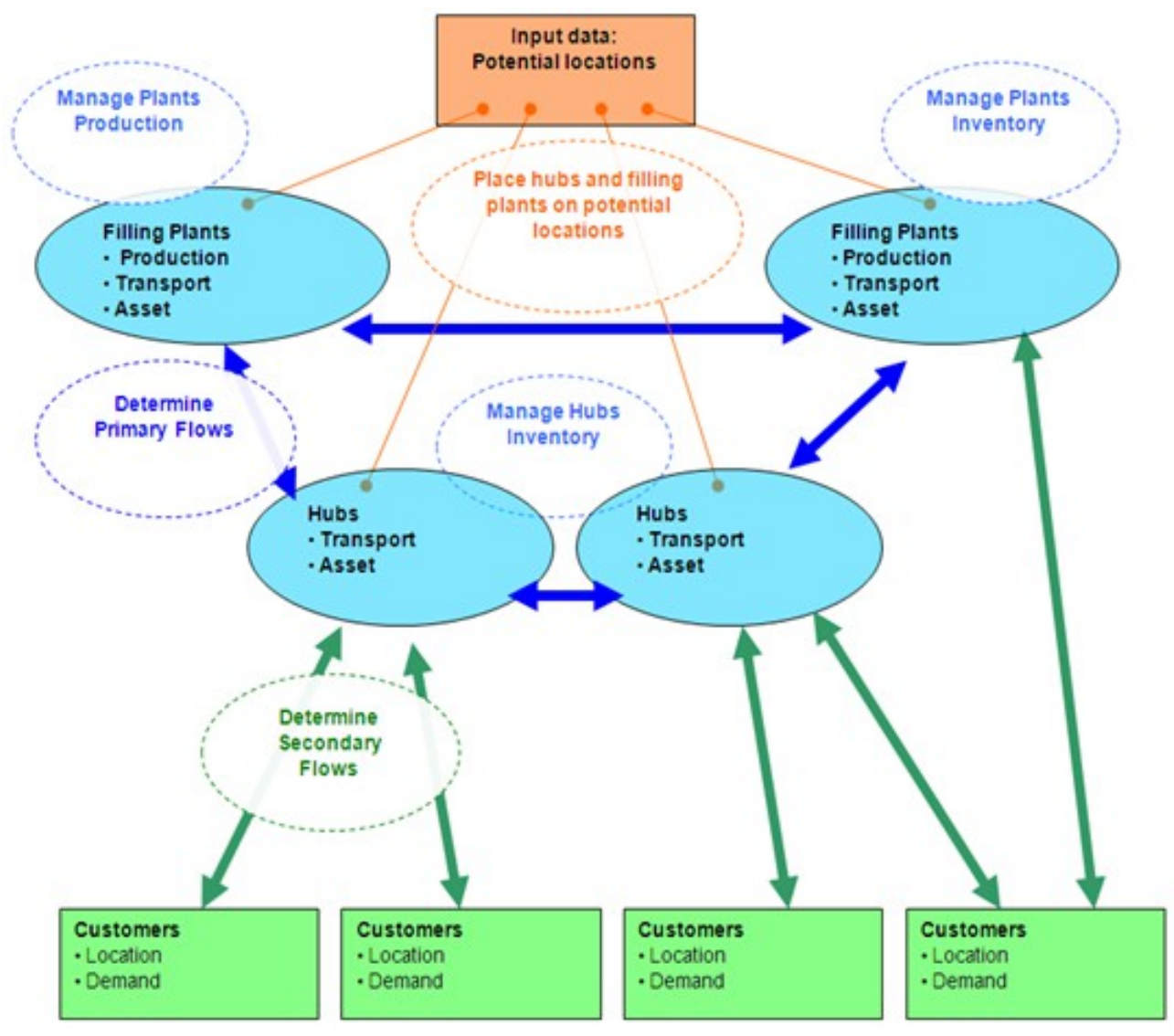

Figure 2. Network Design Problem 
The round trip does not consist of a single delivery but multiple deliveries and this makes the secondary transport cost approximation realistic. For a given customer, an average round trip starting from a given plant is modelled by:

- The driven route split in one dispersion ring which represents the zone where the delivered customers are located and an approach distance to go from the plant to the dispersion ring.

- The average number of customers visited during such a round trip.

- The average number of cylinders delivered during such a round trip.

The radius of the dispersion ring (see Figure 3 ) is set for every customer to a value determined experimentally from real round trips or from round trips generated from a heuristic approach used during the pre-processing of data. The heuristic approach used is not discussed in this paper. The dispersion ring has its centre on the customer under consideration. The approach distance is the shortest distance from the plant to the dispersion ring. Another value found experimentally determines the percentage of customers included in the same dispersion ring which can be delivered in the same round trip. This coefficient aims at correcting the fact that one dispersion ring could withhold several round trips. The secondary round trips have to respect the following constraints:

- The average number of cylinders delivered during the round trip can not exceed the capacity of the trailer used.

- The duration of the round trip can not overcome the maximum driver work time. The round trip duration is mainly a function of the number of customers visited during the trip.

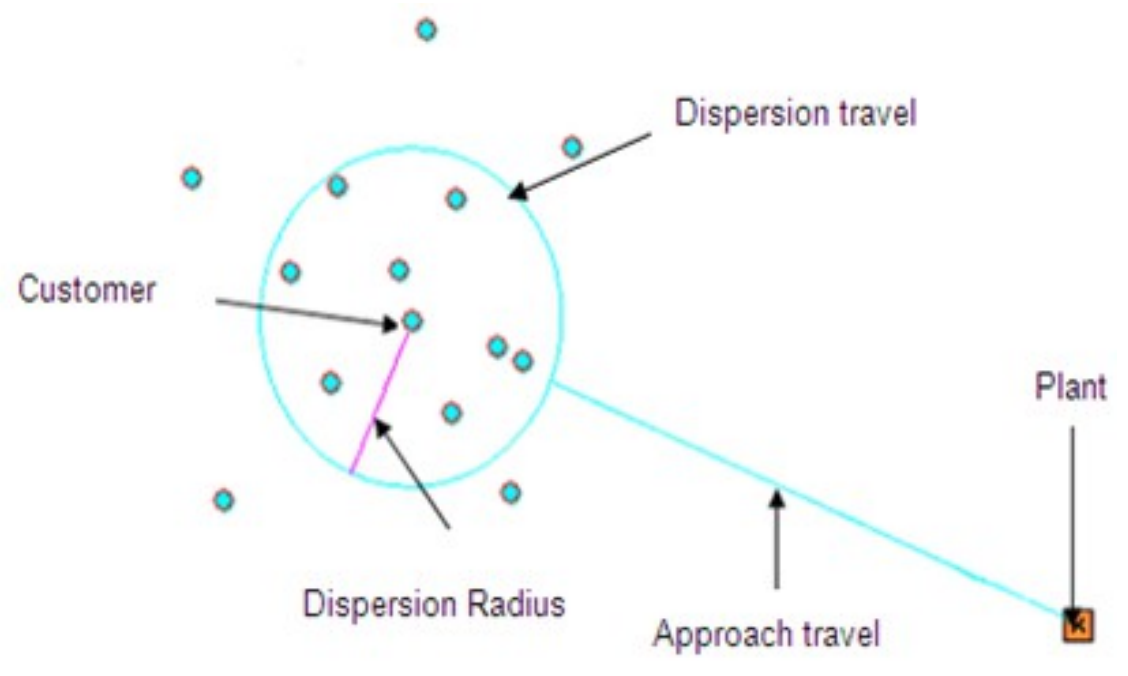

Figure 3. Secondary round trip model 


\subsection{Trucks}

Only two standard trucks are considered in the problem. One truck type is dedicated to primary transport and the other one to the secondary transport. Each type of truck is characterized by its capacity, speed and cost per distance travelled. We assume no limit on the number of trucks of each type that are available for distribution in the model.

\subsection{Inventory Management}

It is important for the cost evaluation of a plant to determine the investment cost necessary for the stocks on its site. The required stock at a plant is composed of:

- Replenishment stock which includes the products filled on the plant everyday and the products delivered from other plants at each primary round trip.

- Delivery stock which represents all the products which are being delivered to customers and other plants every day. When calculating the size of this stock, we assume that the same number of products is delivered every day for this plant.

The stocks take into consideration the variation of demands over a year through a variance of the cylinder flows. The variance of the flows is supposed to be directly proportional to the average volume delivered per day.

\section{Mathematical Model}

To solve the integrated network design problem we propose a mixed integer programming model. In this section we present the main parameters, decision variables and the corresponding mathematical model. We consider the design of a three-tiered supply chain consisting of filling plants, hubs, and customers as described in Section 3. Each customer has deterministic demand. The proposed model provides the needed decisions on how many filling plants and hubs to locate, where to locate them among the list of potential locations, how often to replenish the products at the hubs from the filling plants, what level of working and safety stocks to maintain at the plants, so as to minimize the total system costs consisting of total location, transportation and inventory costs. In other words, the objective is to find the optimal trade-off between transportation costs and all the other costs, mainly the location costs. Inventory costs are a function of the replenishment periods and the demand allocation to the plants.

To simplify the mathematical model, we define two units of measure. We define Equivalent Cylinder (EqCyl) as the unit of area occupied by a 50-litre water capacity compressed gas 
cylinder for transportation on a truck. As the model deals with more than one product, and a truck is allowed to transport many products together, to quantify the capacity of the trucks and also to define the demands of different customers, EqCyl would be used. We also introduce a measure of time called Work Unit (WU). A WU is a unit of time to a physical activity for which time is the main factor to represent work e.g. filling and handling of cylinders. All parameters and variables that denote time are expressed in terms of WU.

\section{Inputs \& Parameters}

\section{I: Set of customers}

J: Set of potential locations

$P$ : Set of products

$T$ : Set of filling tools

$R$ : Set of replenishment periods between plants

$f_{j}$ : Fixed cost (yearly) of locating a filling plant at location $j$, for each $j \in J$

$g_{j}$ : Fixed cost (yearly) of locating a hub at location $j$, for each $j \in J$

$h_{j}$ : Fixed inventory holding cost per EqCyl per year at location $j$, for each $j \in J$

$\chi$ : Fixed cost of a full time employee per year

$\alpha$ : Primary handling productivity at any plant (WU/year/employee)

$\beta$ : Secondary handling productivity at any plant (WU/year/employee)

$\pi_{p}$ : Work time (in $\mathrm{WU}$ ) needed to handle one package of product $p$ at a plant for primary transport, for each $p \in P$

$\theta_{p}$ : Work time (in WU) needed to handle one package of product $p$ at a plant for secondary transport, for each $p \in P$

$W_{p t}$ : Work time (in WU) necessary to fill one package of product $p$ using tool $t$, for each $p \in P$ and $t \in T$

$a_{p}$ : Area (in EqCyl) occupied by one package of product $p$, for each $p \in P$

$m_{t}$ : Filling productivity (WU/employee/year) of a filling tool $t$, for each $t \in T$ 
$b_{t}$ : Fixed cost of using a tool t per year, for each tool $t \in T$

$z_{t}$ : Maximum time (in WU) available to fill packages with tool t per year, for each tool $t \in T$

$S_{p t}$ : Binary parameter, 1 if a filling tool $t$ can fill a package of product $p$, for each $p \in P$ and $t \in$ $T, 0$ otherwise

$\mu_{i p}$ : Average number of packages consumed (yearly) at customer $i$ for product $p$, for each $i \in I$ and $p \in P$

$\sigma_{p}$ : Variance of demand (yearly) for product $p$, for each $p \in P$

$\eta$ : Constant representing number of working days per year (e.g. 250)

$\tau$ : Truck capacity for primary transportation

$c_{r}$ : Average cost per distance travelled during primary transport for a replenishment period $r$, for each $r \in R$

$\lambda_{j i}$ : Average cost per EqCyl from location $j$ to serve customer $i$, for each $j \in J$ and $i \in I$

M: Maximum number of tools at any filling plant

\section{Decision Variables}

$p_{j}$ : Binary variable, 1 if a filling plant is built on location $j$, for each $j \in J, 0$ otherwise

$q_{j}$ : Binary variable, 1 if a hub is built on location $j$, for each $j \in J, 0$ otherwise

$e_{j}$ : Total number of employees working on location $j$, for each $j \in J$

$x_{j p t}$ : Number of packages of product $\mathrm{p}$ filled per year at location $j$ by tool $t$, for each $j \in J, p \in P$, and $t \in T$, a discrete variable

$\Psi_{j t}$ : Number of filling tools of type $t$ required at the location $j$, for each $j \in J$, and $t \in T$, a discrete variable

$u_{j k r}$ : Binary variable, 1 if primary trips are used between locations $j \& k$ after replenish period $r$ such that $j \neq k$, for each $j \in J, k \in J$ and $r \in R, 0$ otherwise

$v_{j k p r}$ : Number of EqCyl of product $p$ delivered from location $j$ to location $k$ during primary trips undergone every replenish period $\mathrm{r}$ such that $j \neq k$, for each $j \in J, k \in J, p \in P$, and $r \in R$ 
$\Phi_{j i}$ : Binary variable, 1 if customer $i$ can be delivered products from location $j$, for each $j \in J, i \in$ $I, 0$ otherwise

$\Omega_{j i p}$ : Number of EqCyl of product $p$ delivered from location $j$ to customer $i$ during secondary trips, for each $j \in J, i \in I, p \in P$

$\omega_{j p}$ : Stock at location $j$ of packages of product $p$, for each $j \in J, p \in P$

The objective function is composed of five main parts as shown below:

- Fixed costs of hubs and filling plants.

- $\quad$ Fixed costs of filling tools.

- Manpower cost dedicated to filling and handling packages.

- Total inventory cost.

- Transport cost, excluding the handling cost at the filling plants and hubs.

Location costs are strategic costs that are incurred when configuring the network. The first two terms in the objective function ensure that fixed costs for either a hub or a filling plant are applied to each selected location. Filling tool costs, manpower costs, and inventory costs are the costs associated with the tactical decisions whereas transport costs are the operational costs. The mathematical formulation of the objective function is given below in Equation (1).

$$
\begin{aligned}
& \text { Minimize } \sum_{j \in J}\left(\left(q_{j}-p_{j}\right) g_{j}+p_{j} f_{j}+\chi e_{j}+\sum_{\substack{k \in J \\
r \in R}} \frac{\eta}{r} u_{j k r} c_{r}+\sum_{\substack{i \in I \\
p \in P}} \lambda_{j i} \Phi_{j i p}+\right. \\
& \left.\sum_{p \in P} a_{p} \omega_{j p} h_{j}+\sum_{t \in T} b_{t} \Psi_{j t}\right)
\end{aligned}
$$

The business constraints which are related to the network structure and flow, primary and secondary transport, and inventory management are given below, (2) - (13):

$$
\begin{gathered}
p_{j} \leq q_{j}, \forall j \in J \\
e_{j} \leq \sum_{\substack{t \in P \\
t \in T}} \frac{w_{p t} x_{j p t}}{m_{t}}+\sum_{k \in J} \sum_{\substack{p \in P \\
r \in R}} \frac{\alpha \pi_{p}}{a_{p}}\left(v_{j k p r}+v_{k j p r}\right)+\sum_{\substack{i \in I \\
p \in P}} \frac{\beta \theta_{p}}{a_{p}} \Omega_{j i p}, \forall j \in J \\
\sum_{p \in P} r \frac{\left(v_{j k p r}+v_{k j p r}\right)}{\eta} \leq \tau u_{j k r}, \forall j \in J, k \in J, r \in R \\
\sum_{r \in R} u_{j j r} \leq 0, \forall j \in J \\
\sum_{r \in R} u_{j k r} \leq 1, \forall j \in J, k \in J
\end{gathered}
$$




$$
\begin{gathered}
\sum_{p \in P} \Omega_{j i p} \leq \Phi_{j i} \sum_{p \in P} a_{p} \mu_{i p}, \forall j \in J, i \in I \\
\sum_{t \in T} \Psi_{j t} \leq p_{j} M, \forall j \in J \\
\sum_{j \in J} w_{p t} x_{j p t} \leq z_{t} s_{p t} \sum_{j \in J} \Psi_{j t}, \forall p \in P, t \in T \\
\sum_{p \in P} w_{p t} x_{j p t} \leq z_{t} \Psi_{j t}, \forall j \in J, t \in T \\
\sum_{j \in J} \Omega_{j i p}=a_{p} \mu_{i p}, \forall i \in I, p \in P \\
\sum_{t \in T} \Psi_{j t} x_{j p t}+\sum_{\substack{k \in J \\
r \in R}} v_{k j p r}=\sum_{i \in I} \Omega_{j i p}+\sum_{\substack{k \in J \\
r \in R}} v_{j k p r}, \forall j \in J, p \in P \\
\omega_{j p}=\sum_{t \in T} \frac{x_{j p t}}{\eta}\left(1+\sigma_{p}\right)+\frac{1+\sigma_{p}}{\eta a_{p}}\left(\sum_{\substack{k \in J \\
r \in R}} \frac{v_{k j p r}}{\eta a_{p}} \max _{\substack{\left.(1, r)+\sum_{k \in J} v_{j k p r}+\sum_{i \in I} \Omega_{j i p}\right) \\
r \in R}}^{\forall j \in J, p \in P}\right.
\end{gathered}
$$

Constraint (2) implies that a filling plant is also a hub. Constraint (3) computes the total number of employees working at a location as a function of different productivities. Constraint (4) puts a maximum limit on the quantity that can be delivered from one location to another depending on the truck capacity used. Constraints (5) and (6) specify that there should not be a primary flow from a location to itself and only one replenishment period between two locations is allowed respectively. Constraint (7) puts a maximum limit on the shipped product quantity in EqCyl from a location to a customer. Constraint (8) requires that filling tools are allocated to locations that are selected for filling plants. Constraint (9) implies that each type of filling tool can only produce a given set of products. Constraint (10) ensures that the total time worked on a tool doesn't exceed its capacity. Constraint (11) specifies that each customer's demand for a given product has to be satisfied. Constraint (12) mentions that on each location and for each product, the number of packages filed on site and received from other locations must allow the total delivery of packages to all customers and other locations. Constraint (13) calculates the stock level at a location for a given product. 


\section{Solution Approach}

The mathematical formulation of network design is a MILP problem. As the traditional facility location problem is NP-complete (Krarup \& Pruzan, 1983), we simplify the model by approximating the routing costs. Moreover, in this paper we are dealing with a real-life largescale problem occurring in packaged gases supply chain. Therefore, we analysed various solving techniques: from near-optimal methods up to approximate ones. The near-optimal approach can be used for small problem instances whereas approximate methods can be applied in the context of large-scale problems. Moreover, we can compare the near-optimal solutions to the approximate ones to benchmark the approximate solutions. In this paper, we provide details about the approximate approaches in order to achieve a reasonable computation time.

\subsection{Mono-Product Approximation}

As the number of products occurring in the packaged gases network design problem implies high complexity, the first approximate approach considered consists of grouping the multiproducts into a single product which we call a mono-product problem. To that aim, each product is treated relatively to its volume of equivalent cylinder (EqCyl) and its type is ignored. Converting multiple product constraints into single product constraints may cause solution infeasibility; the constraints are modified carefully to minimize the likelihood of such infeasibility. As the modified model becomes a single-product model, variables are no longer depending on the number of products available. For example, consider the variable $x_{j p t}$ representing the number of packages of product $\mathrm{p}$ filled per year at location $j$ by tool $t$, for each $j \in J, p \in P$, and $t \in T$. In the mono-product approximation, $x_{j p t}$ is changed to $x_{j t}$ defining the number of EqCyl of the single product filled per year by tool $t$ at location $j$. Similarly, constraints (3), (4), (7), (9), (10), (11), (12) and (13) are modified along with the objective function to represent a single product network design problem.

The resulting problem is also a MILP problem but we do not show the modified model in this paper. The network design problem becomes a unique flow problem and thus, it is easier to solve. We compare the results of this approach with the results obtained by solving the complete model in Section 6. It is shown that this approach gives good solutions especially for placing the hubs' locations and satisfactory results for secondary transport decisions. As this approach does not treat different types of products, the number of filling tools installed at the filling plants is underestimated compared to the optimal solutions. This approach can be used when the problem size is very large and the main interest is to find the network configuration i.e. location of hubs and allocation of customers to hubs whereas resource/inventory 
optimization can be done separately. Figure 4 shows the physical representation of the monoproduct approach.

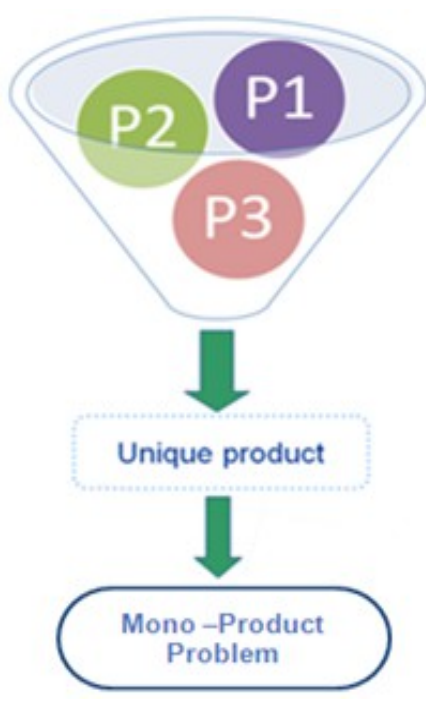

Figure 4. Scheme of Mono-product Approximation

\subsection{Two-steps Decomposition}

In order to reduce the computation time, a typical approach for large-scale problems is based on problem decomposition. We consider a two-step decomposition approach to generate an approximate solution. In the first step of the decomposition approach, the hubs' locations and the hub-customer allocation decisions are determined by solving the mono-product flow problem with minimization of the secondary transportation costs and the hub costs. Secondary transportation cost is more a function of number of cylinders transported between hubs and customers and independent of different products. Therefore it is a safe approximation to determine hubs through optimization of mono-product flow problem. In the second-step, the residual problem is solved based on the multi-product model. The second step optimization determines if the hub built on a given location is a filling plant or not and decides the tools associated with this given filling plant by minimizing the production and the primary transportation costs (tools, sourcing, manpower). Moreover, it optimizes the inventory management by defining the frequency of trips between plants and the flow quantities for the primary transportation. Figure 5 shows the steps in two-step decomposition method and Figure 6 shows the decisions made in each step graphically.

The size of the residual problem in the second step can be further reduced by grouping the products into families of products. The product families are created by selecting the products among the most requested customer's products. Thus, the whole set of products is aggregated 
into families of products. A family essentially is a set of products that can be produced by the same tools. This further reduces the problem size and helps to obtain good results in a reasonable time compared to the complete problem. The grouping does not change the model as it is done in the input data. The second-step model is also an MILP problem and is still hard to be solved to optimality for large-scale network problems. One of the reasons of the complexity to solve the second-step model optimally is that a significant number of binary variables still remain to be optimized for primary transportation.

The MILP mathematical models in our testing are solved on a $2.66 \mathrm{GHz}, 16 \mathrm{~GB}$ RAM server using CPLEX ${ }^{\circledR}$. By tuning the CPLEX parameters, the performance of the CPLEX has been improved on the test cases used. The upper limit on the number of cutting plane passes CPLEX performs when solving the root node of a MILP model is set to 1 . The number of rows in the problem with cuts added is set to 30 times the original number of rows. Relaxation Induced Neighbourhood Search (RINS) heuristic explores the neighbourhood of the current incumbent solution to try to find a new, improved incumbent after every 70 nodes are visited. It is also important to manage the memory problems that occur on a server when solving a large-scale problem. Therefore, the number of stored solutions kept in the solution pool on the server is set to 10. If the node file parameter in CPLEX is set to 0 , when the tree memory limit is reached, optimization is terminated. By setting the node file parameter to 3 , the node files are transferred to disk in compressed form and CPLEX actively manages which nodes remain in memory for processing. An optimality gap has been used for test cases as it is hard to solve the test cases for full optimization. The optimality gap represents the maximum ratio between the optimal solution of the MILP program and its Linear Programming (LP) relaxation.

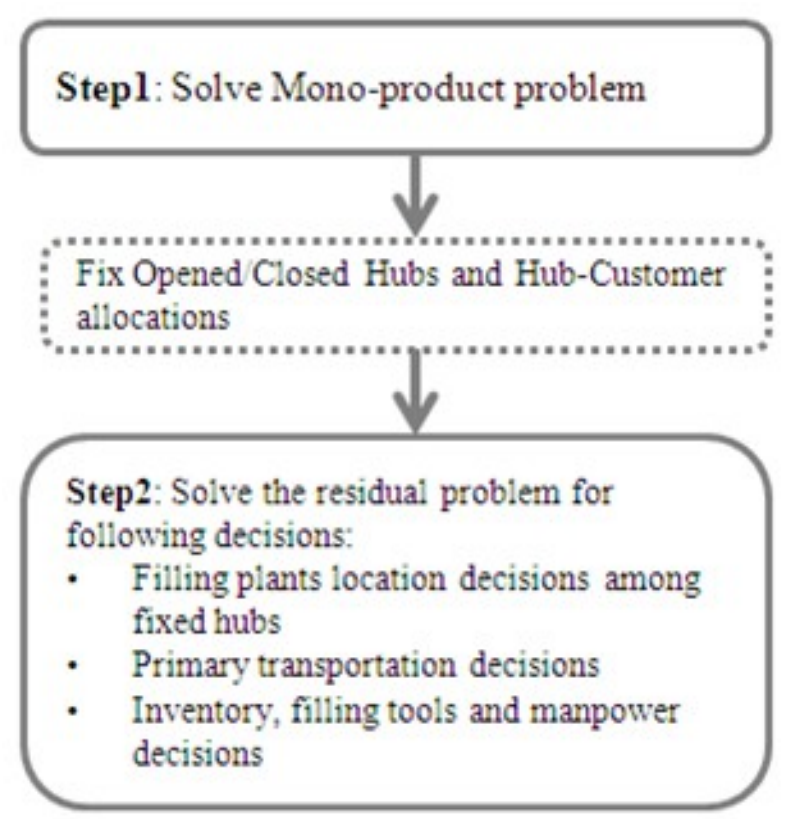

Figure 5. Two-step Decomposition 


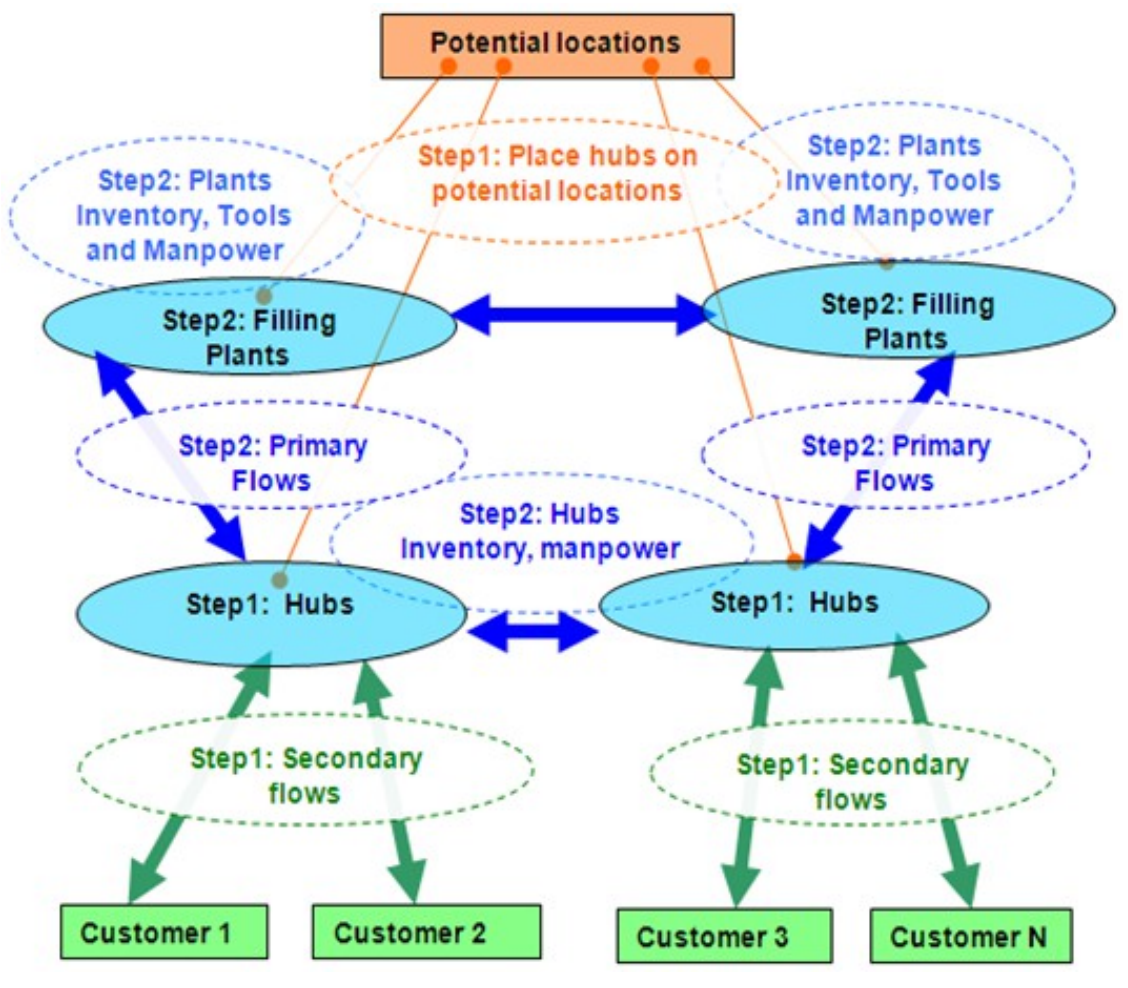

Figure 6. Two-step Decomposition Decisions

\section{Numerical Results}

In this section our objective is to assess the performance of the solution approaches considered in this paper. The proposed solution approaches have been applied to 5 real-life test cases (summarized in Table 1) characterizing the network design problems occurring within the packaged gases distribution networks in different geographical zones. The problem size of a test case is determined mainly by the number of customers, potential locations, types of tools available, and the number of products to be distributed. The given test cases are very different in terms of problem structure and size. This provides a good opportunity to test the approaches for different problem settings and evaluate their scalability.

Each test case in Table 1 has been solved by different approaches providing near-optimal and approximate solutions. It has been observed for the small test cases (e.g. containing up to 2000 customers), a near-optimal solution can be reached in a reasonable computation time (e.g. $60 \mathrm{~min}$ for test case 2). For test cases 1 and 2, the facility location decisions i.e. the number and the set of locations to be opened as filling plants/hubs from the mono-product and the two-steps approaches are exactly the same as from the near-optimal solution. This shows that both the approximate approaches are successful in determining optimal strategic decisions. In the test results, mono-product approach underestimates the total network cost which is expected due to the simplification of the model. Therefore, we do not consider monoapproach for tactical and operational decisions as it solves an approximate model. For test case 
1, two-steps approach provides the same network cost as from the near-optimal method with the same optimality gap. For test case 2, the two-steps approach provides a solution with $1.2 \%$ higher network cost than the near-optimal solution. For test case 3, the near-optimal solution was not generated as we could not solve the complete problem within an acceptable optimality gap. For real-life network design, we believe that the computation time in a few hours is acceptable due to the fact that the opportunities to setup a new supply chain network or completing overhauling an existing one are not very frequent. The computation time for test cases 1 and 2 with the two-steps is relatively small but test case 3 takes more than 15 hours to obtain a solution within the optimality gap of $0.43 \%$. It is also possible to achieve a solution in few hours by increasing the optimality gap to $5 \%$ as shown in the Table 2 . For test case 3 , the benchmarking of two-steps decomposition solution is done by comparing the facility location decisions with a manual solution based on the network designer's experience. The gap analysis with the manual solution shows that the two-steps solution for test case 3 provides a solution with total network cost which is $6.4 \%$ less than the manual solution. Test cases $4 \& 5$ are similar in problem size to test case 3 and therefore, we have obtained solutions from mono-product and two-steps approaches only. Cost deviation (\%) provides the total yielded cost deviation from the near optimal solution. If near optimal solution is not available, the cost deviation is calculation from the mono-product method. These two test cases are also benchmarked using manual solutions. Two-steps approach provides an average savings of $5.5 \%$ and $5.8 \%$ for test cases $4 \& 5$ respectively.

\begin{tabular}{|c|c|c|c|c|}
\hline Instance & Customers & Potential Locations & Tools & Products \\
\hline 1 & 520 & 4 & 3 & 5 \\
\hline 2 & 1,964 & 14 & 6 & 4 \\
\hline 3 & 12,036 & 26 & 22 & 43 \\
\hline 4 & 12,345 & 30 & 18 & 35 \\
\hline 5 & 13491 & 23 & 24 & 41 \\
\hline
\end{tabular}

Table 1. Real-life network design test cases 


\begin{tabular}{|c|c|c|c|c|c|c|c|}
\hline Test Case & Gap (\%) & Solution Approach & $\begin{array}{l}\text { Solver Time } \\
\qquad(\mathrm{min})\end{array}$ & Total Cost & $\begin{array}{c}\text { Cost Deviation } \\
(\%)\end{array}$ & Filling Plants & Hubs \\
\hline \multirow{3}{*}{1} & \multirow{3}{*}{0.5} & Mono-product & 2 & 561,891 & -28.1 & 2 & 3 \\
\hline & & Two-Steps & 6 & 782,070 & 0 & 2 & 3 \\
\hline & & Near-Optimal & 4 & 782,070 & 0 & 2 & 3 \\
\hline \multirow{3}{*}{2} & \multirow{3}{*}{0.5} & Mono-product & 11 & $2,147,515$ & -6.8 & 6 & 11 \\
\hline & & Two-Steps & 593 & $2,303,703$ & 1.2 & 6 & 12 \\
\hline & & Near-Optimal & 63 & $2,276,267$ & 0 & 6 & 12 \\
\hline \multirow{3}{*}{3} & 0.5 & Mono-product & 121 & $19,289,480$ & 0 & 11 & 18 \\
\hline & 5.0 & Two-Steps & 332 & $24,125,572$ & 25.1 & 11 & 18 \\
\hline & 0.43 & Two-Steps & 948 & $23,160,386$ & 20.1 & 11 & 18 \\
\hline \multirow{2}{*}{4} & 0.5 & Mono-product & 141 & $18,927,632$ & 0 & 15 & 20 \\
\hline & 5.0 & Two-Steps & 383 & $25,840,433$ & 36.5 & 15 & 23 \\
\hline \multirow{2}{*}{5} & 0.5 & Mono-product & 154 & $23,082,110$ & 0 & 9 & 15 \\
\hline & 5.0 & Two-Steps & 401 & $29,733,484$ & 28.8 & 10 & 16 \\
\hline
\end{tabular}

Table 2. Performance Test Results

Figure 7 shows the different cost components as percentages of the total network design cost for all test cases solved with two-steps approach with an optimality gap of $5 \%$. Figure 7 shows that the facility location costs and the secondary transportation costs are the highest cost components of the total network cost and therefore, have more influence on the network design decisions. Since the network design model studied in this paper is deterministic, we also perform sensitivity analysis to check the impact of different parameters on the facility location and other network decisions. The parameters which are more likely to change over time are demand, unit transportation cost, and manpower cost.

Figure 8 shows the location decisions of scenarios obtained by changing the demand at each customer equally for test case 2 which is solved with near-optimal approach. The results show that the facility location decisions i.e. the number and the set of opened facilities do not change even with more than $5 \%$ increase or decrease in demand at each customer location. The main reason for such stability is that we increased the product demand equally for all the customers to perform sensitivity analysis. In reality, the demand of different customers does not homogenously increase or decrease over time. Also, the impact of demand change on inventory and transportation costs (both primary and secondary) is more compared to the other costs. When facility location decisions don't change with modified demand, the change in inventory and transportation costs is nearly linear with demand change. Table 3 provides similar sensitivity analysis results for unit transportation cost and manpower cost for test case 2. Manpower is mainly a function of demand and therefore, does not influence facility location decisions significantly as evident from the results. 


\begin{tabular}{|c|c|c|c|c|}
\cline { 2 - 5 } \multicolumn{1}{c|}{} & \multicolumn{2}{c|}{ Unit Transportation Cost } & \multicolumn{2}{c|}{ Manpower Cost } \\
\hline$\%$ Change & Filling Plants & Hubs & Filling Plants & Hubs \\
\hline$-20.0 \%$ & 4 & 10 & 7 & 13 \\
\hline$-15.0 \%$ & 4 & 11 & 6 & 12 \\
\hline$-10.0 \%$ & 5 & 11 & 6 & 12 \\
\hline$-5.0 \%$ & 6 & 12 & 6 & 12 \\
\hline $0.0 \%$ & 6 & 12 & 6 & 12 \\
\hline $5.0 \%$ & 6 & 13 & 6 & 12 \\
\hline $10.0 \%$ & 7 & 14 & 6 & 12 \\
\hline $15.0 \%$ & 7 & 14 & 6 & 12 \\
\hline $20.0 \%$ & 7 & 14 & 5 & 11 \\
\hline
\end{tabular}

Table 3. Cost Sensitivity Analysis for test case 2

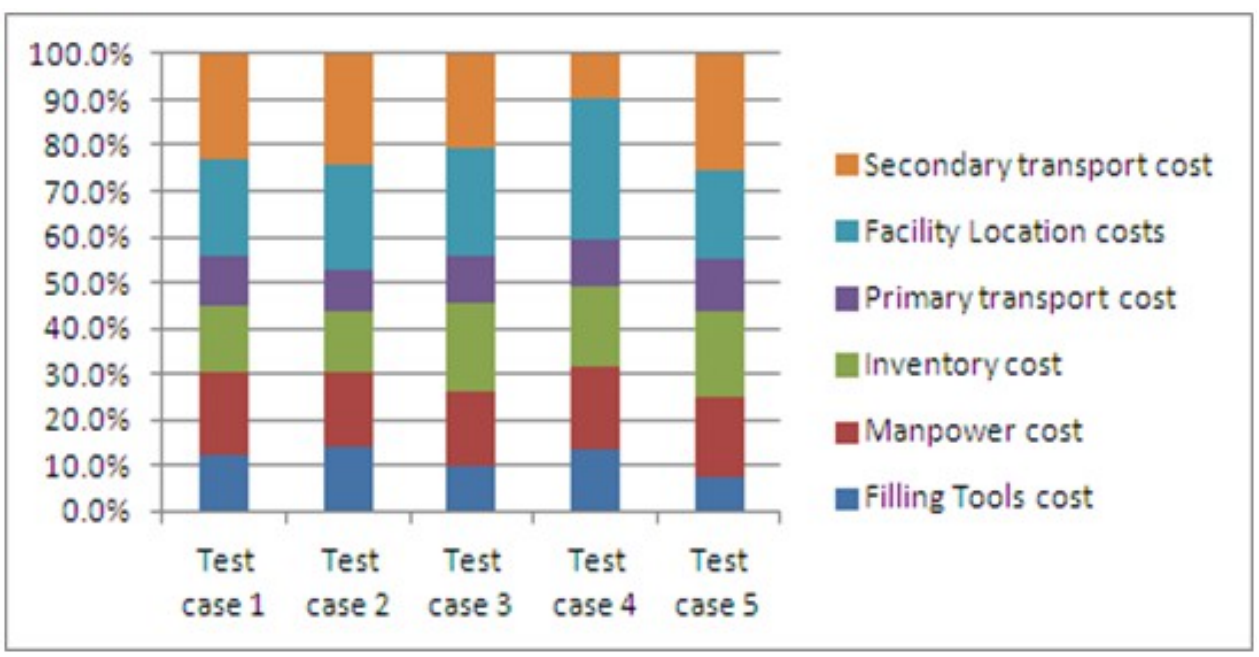

Figure 7. Network Design Costs for 3 test cases

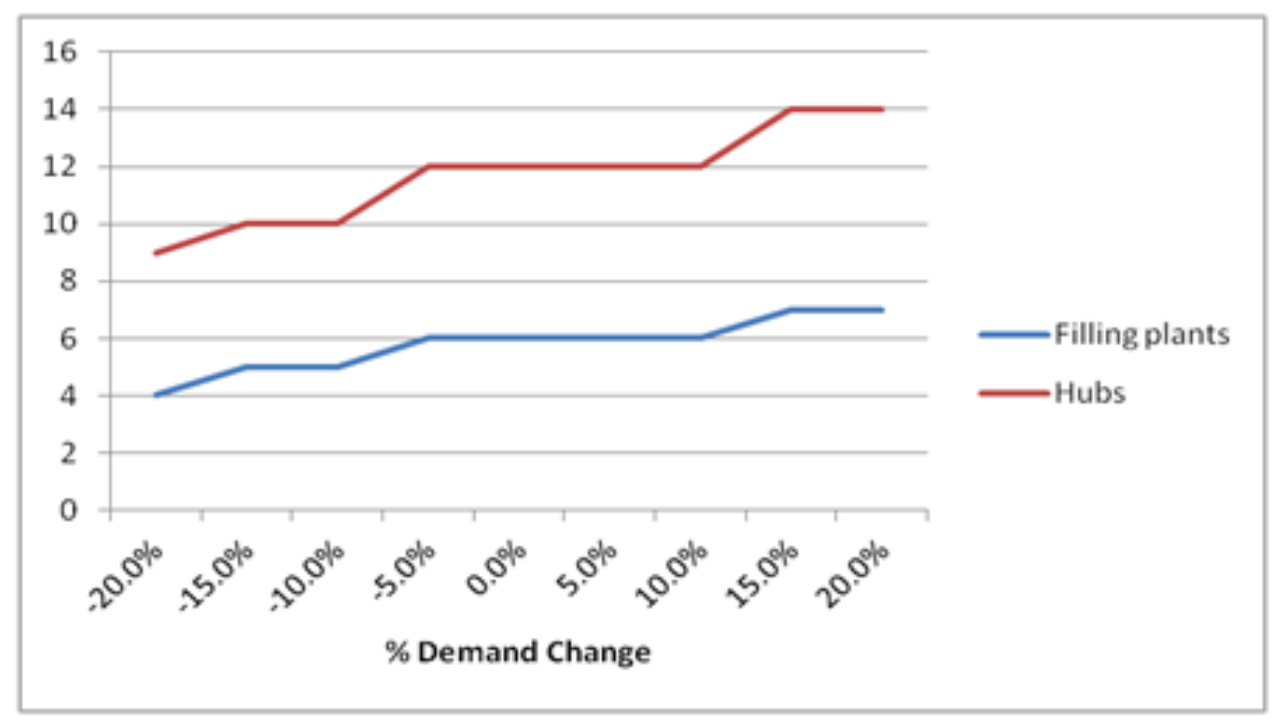

Figure 8. Demand Sensitivity Analysis for test case 2 
For test case 2, manpower costs have to increase or decrease at least $20 \%$ to make a change in the facility location decisions. Since the primary and the secondary transportation costs represent a significant portion of network design costs, the facility location decisions are sensitive to the unit transportation cost. An increase in the unit transportation cost causes more hubs to open along with filling plants which is expected to minimize the secondary transportation costs. We also perform sensitivity analysis of the assets like filling tools needed at a particular location. We perform such an analysis for test case 3 with respect to filling tools called Type-F. Type-F filling tools have a high productivity as they are completely automated and don't require human intervention to mix different gases. The operator has to only specify the specification of the end-product and Type- $\mathrm{F}$ tool would fill the required percentages of component gases to produce the final product. We use 6 different varieties of Type- $F$ tools based on the component gases they can mix. Type- $F$ tools generally cost more than the manual filling tools that produce the same type of products but are less time-efficient due to required human intervention. We also perform sensitivity analysis to check whether the decision to install Type- $F$ tools changes when some systems parameters like payback period are modified. Table 4 shows 5 parameters with standard values and possible potential values.

\begin{tabular}{|c|c|c|}
\cline { 2 - 3 } \multicolumn{1}{c|}{} & Standard & Potential Value \\
\hline Primary Truck Capacity & $260 \mathrm{EqCyl}$ & $240 \mathrm{EqCyl}$ \\
\hline Cylinder Cost & 30 & 25 \\
\hline Primary Transport Cost & 1.2 & 1.38 \\
\hline Primary Handling Productivity & 25,000 & 22,000 \\
\hline Payback Period of Type-F tools & 3 years & 2 years \\
\hline
\end{tabular}

Table 4. Parameter Values

Figure 9 shows the allocation of 6 varieties of Type- $F$ tools in the optimal solution for test case 3 solved with standard parameters. Note that we had 11 fillings plants in the optimal solution of original test case 3. To validate the robustness of the allocation of Type- $F$ tools to various locations, we perform sensitivity analysis for 5 parameters in Table 4 with their potential values. When payback period is changed to 2 years, we get a completely different solution as shown in Figure 10. The changed solution is due to the reason that with the decrease in payback period, the annual savings have to be more to justify the investment in the Type- $F$ tools. The results show that the decision to invest into Type- $F$ tools is sensitive to all 5 parameters which are summarized in Table 5. 


\begin{tabular}{|c|c|c|c|c|c|c|c|}
\cline { 2 - 8 } \multicolumn{1}{c|}{} & Loc. $\mathbf{1}$ & Loc. 2 & Loc. 6 & Loc. 9 & Loc. $\mathbf{1 0}$ & Loc. $\mathbf{1 1}$ & Total Cost \\
\hline Standard Parameters & $\mathrm{X}$ & $\mathrm{X}$ & $\mathrm{X}$ & $\mathrm{X}$ & $\mathrm{X}$ & $\mathrm{X}$ & $23,070,000$ \\
\hline Primary Truck Capacity $=240$ & & $\mathrm{X}$ & $\mathrm{X}$ & $\mathrm{X}$ & $\mathrm{X}$ & $\mathrm{X}$ & $23,230,000$ \\
\hline Cylinder cost $=25$ & & $\mathrm{X}$ & & & & & $22,980,340$ \\
\hline Primary Transport Cost $=1.38$ & $\mathrm{X}$ & $\mathrm{X}$ & $\mathrm{X}$ & $\mathrm{X}$ & $\mathrm{X}$ & $\mathrm{X}$ & $23,560,000$ \\
\hline Primary WU $=22,000$ & & $\mathrm{X}$ & $\mathrm{X}$ & $\mathrm{X}$ & $\mathrm{X}$ & & $23,380,000$ \\
\hline Payback $=2$ years & & $\mathrm{X}$ & $\mathrm{X}$ & & & & $23,100,000$ \\
\hline
\end{tabular}

Table 5. Type-F Filling tools Sensitivity Analysis

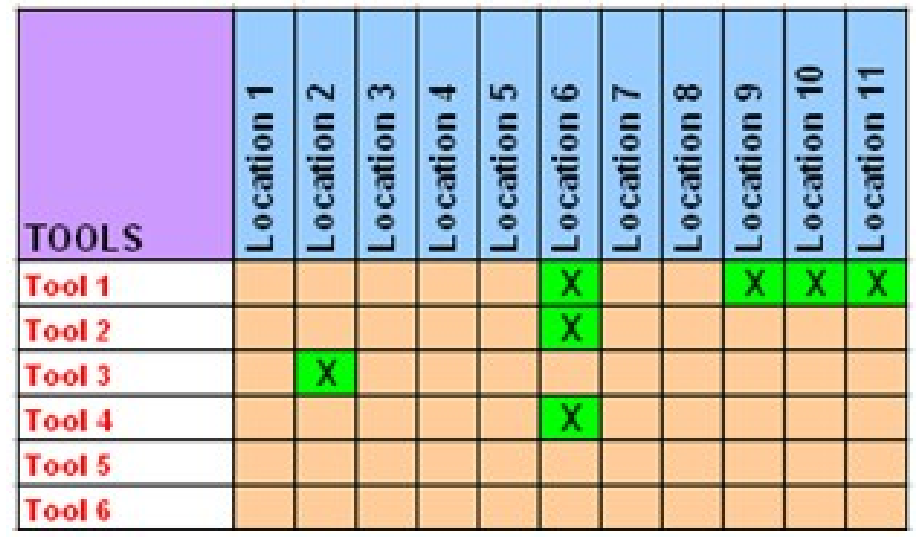

Figure 9. Type-F Filling Tools Allocation

\begin{tabular}{|c|c|c|c|c|c|c|c|c|c|c|c|}
\hline TOOLS & 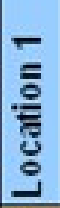 & 을 & m & 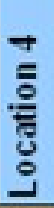 & 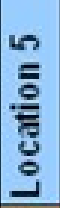 & 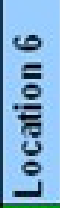 & 들 & 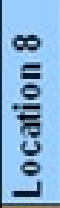 & 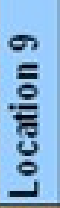 & 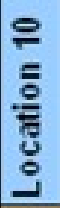 & 등 \\
\hline Tool 1 & & & & & & $\mathrm{X}$ & & & & & \\
\hline \multicolumn{12}{|l|}{ Tool 2} \\
\hline Tool 3 & & $x$ & & & & & & & & & \\
\hline \multicolumn{12}{|l|}{ Tool 4} \\
\hline \multicolumn{12}{|l|}{ Tool 5} \\
\hline Tool 6 & & & & & & & & & & & \\
\hline
\end{tabular}

Figure 10. 2 Years Payback period Solution

In the first scenario, the reduction in the truck capacity causes a loss in the primary transport efficiency: in order to deliver the same quantity of products more transport is needed. In that way, primary transport cost increases and to compensate for this, the solution favors the local production. When cylinder cost decreases, the overall cost including production, stock, handling and transport are reduced. Therefore, the solver is comparing the profitability of installing Type- $F$ tools with the depreciation cost of tools. According to the results provided, the primary transport is favored instead of production, hence the small number of tools added. In the third scenario, the production is reduced and thus, the primary handling productivity is 
decreasing and more work has to be done to satisfy the same production demand. The result recommends adding several filling tools in order to reduce the primary transport and thus, the primary handling activities. When fuel price increases transport cost rises as well so in order to avoid transport cost, the solver takes advantage of installing many tools and develops local production. By comparing the results on all scenarios, and analyzing where and which production tools are frequently installed by solver, a final recommendation on new assets investment is made. It consists of recommending at which locations more filling tools should be added in order to reduce the total costs.

\section{Conclusions}

In practice supply chain network configuration typically involves optimizing strategic decisions without considering their impact on all the tactical delivery planning and daily scheduling decisions. In this paper we optimize not only strategic decisions but also consider all tactical and operational decisions in the mathematical model for the network configuration. We specifically consider the integrated network design problem dedicated to the packaged gases distribution. The main goals for solving the integrated network design problem include determining the locations of the hubs and the filling plants, the production capacity of the filling plants, the primary and the secondary cylinders flows and the inventory of both the filling plants and the hubs. To solve it, we propose a mathematical model which combines both the location-routing and the location-inventory integrated models and approximates the routing cost used in both the integrated models. In order to solve real large-scale problems, we propose approximate decomposition based approach. We applied near-optimal and approximate approaches on 3 real-life test cases from packaged gases cylinder distribution. The obtained solutions are within an acceptable optimality gap from the optimal solutions. The results indicate that mono-approach and two-steps approaches are capable to generate good facility location solutions in a reasonable time and are comparable to near-optimal solutions on smaller test cases. The difference between mono-product and two-steps is that two-steps method provides a better estimate of tactical and operational costs. For large-scale test cases, it is hard to obtain near-optimal solutions whereas two-steps approximation can generate good solutions in an acceptable time. Therefore, near-optimal approach is suitable for smaller test cases and approximation approaches for large-scale test cases.

In the future, further studies on improvements relative to the computation time to solve the complete model without using decomposition approach can be envisioned. Also, further research can be done to benchmark the approximate approaches considered in this paper for large-scale test cases. Even though we performed sensitivity analysis for few input parameters, future work can be focussed on developing and solving a robust model for packaged gases network design. 


\section{References}

Adelman, D. (2003). A price-directed approach to stochastic inventory/routing. Working Paper, University of Chicago.

Balakrishnan, A., Ward, J.E., \& Wong, R.T. (1987). Integrated facility location and vehicle routing models: recent work and future prospects. American Journal of Mathematical and Management Sciences, 7, 35-61. http://dx.doi.org/10.1080/01966324.1987.10737207

Crainic, T.G., \& Laporte, G. (1997). Planning models for freight transportation. European Journal of Operations Research, 97, 409-438. http://dx.doi.org/10.1016/S0377-2217(96)00298-6

Daskin, M.S., \& Owen, S.H. (1999). Location models in transportation. In Handbook of Transportation Science (Eds R. Hall), 311-360. http://dx.doi.org/10.1007/978-1-4615-5203-1_10

Erlebacher, S.J., \& Meller, R.D. (2000). The interaction of location and inventory in designing distribution systems. IIE Transactions, 32, 155-166. http://dx.doi.org/10.1080/07408170008963888

Kleywegt, A., Nori, V.S., \& Savelsbergh, M.W.P. (2002). The stochastic inventory routing problem with direct deliveries. Transportation Science, 36, 94-118.

http://dx.doi.org/10.1287/trsc.36.1.94.574

Klose, A., \& Drexl, A. (2005). Facility location models for distribution system design. European Journal of Operational Research, 162, 4-29. http://dx.doi.org/10.1016/j.ejor.2003.10.031

Krarup, J., \& Pruzan, P.M. (1983). The simple plant location problem-survey and synthesis. European Journal of Operational Research, 2, 36-81. http://dx.doi.org/10.1016/0377-2217(83)90181-9

Melo, M., Nickel, S., \& Saldanha-da-Gama, F. (2009). Facility location and supply chain management - a review. European Journal of Operations Research, 196(2), 401-412. http://dx.doi.org/10.1016/j.ejor.2008.05.007

ReVelle, C., \& Eiselt, H. (2005). Location analysis: a Synthesis and survey. European Journal of Operational Research, 165, 1-19. http://dx.doi.org/10.1016/j.ejor.2003.11.032

Shen, Z.M. (2000). Approximation Algorithms for Various Supply Chain Problems. PhD Thesis. Department of Industrial Engineering and Management Sciences, Northwestern University.

Journal of Industrial Engineering and Management, 2015 (www.jiem.org)

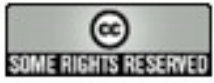

Article's contents are provided on a Attribution-Non Commercial 3.0 Creative commons license. Readers are allowed to copy, distribute and communicate article's contents, provided the author's and Journal of Industrial Engineering and Management's names are included. It must not be used for commercial purposes. To see the complete license contents, please visit http://creativecommons.org/licenses/by-nc/3.0/. 